\section{VIVIENDA SOCIAL EN CÓRDOBA: EFECTOS EN LA SEGREGACIÓN RESIDENCIAL Y EL CRECIMIENTO URBANO $(1991-2008)^{1}$}

María Cecilia Marengo² y Ana Laura Elorza ${ }^{3}$

\section{SOCIAL HOUSING IN CORDOBA, CONSEOUENCES ON RESIDENTIAL SEGREGATION AND URBAN GROWTH (1991-2008)'}

\author{
María Cecilia Marengo², Ana Laura Elorza ${ }^{3}$
}

\section{Abstract}

This paper analyzes the role of the State in the production and reproduction of residential habitat through the implementation of housing policies. Background studies are reviewed that account for the relationship between social housing, location and its effects on the spread of urban growth and residential segregation. Subsequently, this research analyzes the evolution of residential segregation in Córdoba over the 1991-2008 period and discusses the impact of social housing programs on the dynamics of such a phenomenon. In other words this paper studies the effects of recent State programs implemented in Cordoba over the last decades which, 
las últimas décadas en la ciudad de Córdoba, que si bien proveen el bien (vivienda), al localizarse en áreas periféricas dificultan a sus destinatarios el acceso a las ventajas en términos de centralidad (bienes y servicios), así como las posibilidades de contacto con un medio social diverso y variado. Finalmente, se concluye con reflexiones y consideraciones para tener en cuenta en la futura formulación de proyectos de hábitat social.

\section{PALABRAS CLAVE: POLÍTICA DE VIVIENDA; SEGREGACIÓN RESIDENCIAL; EXTENSIÓN URBANA.}

Recibido: 15-06-2015

Aceptado: 18-01-2016 while providing goods (dwellings), their location in peri-urban areas has prevented inhabitants from accessing the advantages of centrality (provision of goods and services) and interacting with a diverse and varied environment. Finally, this research offers some reflections on the analysis and provides considerations that should be taken into account in the formulation of future social housing projects.

\section{KEYWORDS: HOUSING POLICY, RESIDENTIAL SEGREGATION, URBAN ENCROACHMENT.}

Received: 15-036-2015

Accepted: 18-01-2016
1 Artículo realizado a partir de los resultados de investigaciones financiadas por el Consejo Nacional de Investigaciones Científicas y Técnicas (CONICET) de Argentina y por la Secretaría de Ciencia y Técnica de la UNC (SECyT), "La expansión urbana, estrategias de intervención en contextos de alta inequidad socio-espacial" y "Segregación residencial socioeconómica en la ciudad de Córdoba 2001- 2008. Dinámica y efectos en la vida cotidiana de la población pobre segregada", desarrolladas en el período 2010-2014.

2 Argentina. Investigadora independiente del Consejo Nacional de Investigaciones Científicas y Técnicas (CONICET). Profesora titular de la Facultad de Arquitectura, Urbanismo y Diseño de la Universidad Nacional de Córdoba. Correo electrónico: mcmarengo@gmail.com.

3 Argentina. Investigadora del Instituto de Investigación de la Vivienda y Hábitat de la Facultad de Arquitectura, Urbanismo y Diseño de la Universidad Nacional de Córdoba. Becaria de CONICET. Correo electrónico: analauraelorza@hotmail.com.
1 This paper is based on the results of research entitled "Urban Growth, Intervention Strategies Within High Socio-Spatial Inequality Contexts" and "Socioeconomic Residential Segregation in Cordoba, 2001-2008. Dynamics and Effects on the Daily Lives of Low-Income Segregated People", both conducted between 2010 and 2014 and funded by the National Scientific and Technical Research Council (CONICET), Argentina, and the Department of Science and Technology (SECyT) at the National University of Cordoba.

2 Argentina. Independent researcher, National Scientific and Technical Research Council (CONICET). Professor, Faculty of Architecture, Urban Planning and Design, National University of Cordoba. Email: mcmarengo@gmail.com

3 Argentina. Researcher, Housing and Habitat Institute, Faculty of Architecture, Urban Planning and Design, National University of Cordoba. CONICET fellow. Email: analauraelorza@hotmail. com 


\section{Introducción}

En la década de los noventa, a partir de la constatación de los impactos de la reestructuración del modelo de acumulación capitalista en las estructuras urbanas, el estudio de la segregación residencial toma una nueva relevancia. Distintos investigadores destacan la profundización de la segregación residencial como rasgo de las metrópolis en los últimos tiempos ${ }^{4}$. En América Latina, a pesar que las ciudades se caracterizaron desde su conformación por ser segregadas ${ }^{5}$ el análisis de este fenómeno adquiere importancia asociado a los cambios económicos y la implementación de políticas de corte neoliberal que se producen en la última década del siglo XX; se señala que estaría creciendo la escala geográfica e intensificándose las consecuencias negativas en la calidad de vida material y simbólica de la población pobre segregada ${ }^{6}$.

Existe un creciente consenso por parte de estudiosos y formuladores de políticas urbanas, acerca de cómo la localización en la ciudad se relaciona con el acceso a las oportunidades y ventajas derivadas de la aglomeración. "El lugar donde se habita tiene una importancia central: una mala localización, si se suma al desempleo, la pobreza y el déficit de

4 Harvey, 2000; Davis, 2006 y Borja, 2007, entre otros.

5 Katzman, 2001; Duhau, 2013 y Sabatini, 2004.

6 Sabatini, Cáceres y Cerda, 2001; Rodríguez y Sugranyes, 2005; Brain y Sabatini, 2006. servicios y transporte público, es co-determinante en la falta de oportunidades. El conjunto de carencias socio-urbanas presentes en determinadas áreas exceden las desigualdades sociales y de ingresos, para convertirse en un mecanismo que contribuye a reproducir la desigualdad y la marginalización económica y social”’

El Estado participa en la producción / reproducción de la segregación residencial; lo hace estableciendo condiciones de parcelamiento, edificabilidad y tipo de construcción, a través de la normativa urbana o de acciones directas de localización de proyectos de vivienda social. Generalmente, los mismos se ubican en áreas periféricas donde los precios del suelo son bajos por su condición de bordes como por los déficits que presentan en términos de provisión de infraestructura, servicios y equipamientos necesarios para la reproducción social.

Los efectos negativos de la segregación se relacionan con su carácter involuntario. Los grupos pobres son excluidos de ciertos sectores de la ciudad y empujados a aglomerarse en las peores áreas.

Las políticas de vivienda social (y específicamente los programas de construcción de nuevos barrios) están condicionadas por la estructura de los precios de suelo urbano y las restricciones

7 Cuenya, 2014, p. 107. 
presupuestarias. Dado que los proyectos deben ajustarse a determinadas condiciones y valores finales asignados a la materialización de los proyectos (establecidos en cada operatoria), el precio del terreno y los metros cuadrados de superficie residencial son las variables de ajuste con mayor incidencia en la ecuación económica. Estudios realizados en diferentes ciudades de la región latinoamericana identifican como rasgo constante de las políticas de vivienda social implementadas desde la década de los noventa, la localización cada vez más periférica de los proyectos habitacionales (asociada al menor valor del suelo). Esta condición ha devenido en profundos problemas para la reproducción social de los sectores de bajos ingresos. El aumento de las distancias respecto de los lugares de servicio, aprovisionamiento y trabajo, la desestructuración de las redes sociales primarias y secundarias como consecuencia de la localización en los bordes y la construcción de territorios socialmente homogéneos con reducidas posibilidades de interacción entre grupos sociales diversos, son algunos de los efectos derivados ${ }^{8}$.

Córdoba (Argentina), al igual que la mayoría de las ciudades, se caracteriza por una estructura de desigual apropiación del espacio urbano según la condición socioeconómica de los grupos sociales; la mayoría de la población de bajos ingresos habita las áreas periféricas, históricamente conformadas con menor oferta de servicios, equipamientos sociales e infraestructura urbana. En trabajos previos $^{9}$ hemos abordado el estudio de la segregación residencial socioeconómica, identificando áreas segregadas en términos de localización de población con necesidades básicas insatisfechas (NBI), en los cortes temporales 1991-2001 y hemos estudiado la evolución de las tendencias de este fenómeno ampliando la periodización y los atributos considerados en el análisis. Otros trabajos desarrollados en Córdoba ${ }^{10}$ analizan esta problemática desde la perspectiva de la concepción de las políticas públicas.

En este artículo nos interesa avanzar en la comprensión de las relaciones entre las políticas de vivienda social y la producción de la segregación residencial socioeconómica, efectivamente medida en el espacio territorial. La hipótesis que sostenemos es que los proyectos de vivienda social "llave en mano" (que el Estado ha desarrollado en las últimas décadas en la ciudad de Córdoba) han intensificado el fenómeno. Como consecuencia de la política habitacional, determinadas áreas urbanas se constituyen hoy en sectores críticos, donde se reproducen condiciones de pobreza y marginación.

9 Marengo, 2004; Marengo y Elorza, 2014.

10 Tecco y Fernández, 2005.
8 Rodríguez y Sungranyes, 2005; Brain y Sabatini, 2006; Hidalgo, 2007. 
La primera sección del artículo presenta una breve descripción del proceso de crecimiento urbano caracterizado por la expansión en baja densidad y sus efectos en la segregación residencial socioeconómica. La segunda sección aborda el concepto y las dimensiones de análisis desde una perspectiva teórica latinoamericana. En tercer lugar, se desarrolla una exploración de los antecedentes de estudios que dan cuenta de la relación entre políticas de vivienda social, localización y segregación residencial. Posteriormente, se analiza la evolución del fenómeno en Córdoba en el periodo 1991-2008 y los efectos de los programas de vivienda social en la dinámica del mismo. Finalmente, se concluye con reflexiones para la futura formulación de proyectos de hábitat social.

\section{EL CRECIMIENTO URBANO: HACIA UNA CIUDAD DISPERSA}

Los estudios sobre la evolución del crecimiento urbano en las principales ciudades de la región así como en ciudades de países desarrollados, señalan que el rasgo característico en las últimas décadas es la extensión de la urbanización. La tendencia generalizada es hacia la metropolización expandida, la conformación de áreas metropolitanas con bordes difusos y de alcance regional y la declinación de las densidades en la ocupación del espacio habitado ${ }^{11}$. Se constata que la anexión de suelo urbanizable acompaña las transformaciones urbanas producidas en las últimas décadas, aun cuando el crecimiento demográfico se ha desacelerado.

El incremento de la cantidad de población que vive en los suburbios, la descentralización de actividades hacia la periferia y la materialización de grandes proyectos comerciales, recreativos y residenciales en los bordes de la ciudad, producen un desarrollo con mayor dispersión física y funcional en la estructura urbana. La expansión significativa de la forma urbana (comparativamente con la etapa de industrialización) se atribuye a la menor intervención del Estado en la regulación del crecimiento, la flexibilización en las normativas para facilitar la inversión privada y la creciente polarización en la estructura social.

A partir de la década del noventa se constata, en la mayoría de las ciudades de la región, que las formas socio-espaciales del espacio periférico se asocian con una ciudad más dividida. Las desigualdades sociales -rasgo característico de la urbanización en Latinoamérica- se manifiestan en la conformación de un espacio residencial dual y polarizado, con entornos residenciales de lujo físicamente diferenciados y simultáneamente, áreas empobrecidas que concentran población con alta vulnerabilidad

11 ONU HÁBITAT 2012; Ángel, Parent, Civco y Blei, 2010. 
social y bajos niveles de inversión pública en equipamiento urbano y obras de infraestructura.

La extensión del crecimiento físico responde a diferentes lógicas de producción del espacio que compiten por un recurso escaso, el suelo urbano: la lógica del capital privado, la lógica de la acción del Estado y la lógica de la necesidad ${ }^{12}$ (de aquellos habitantes que no acceden a la vivienda por el mercado formal y lo hacen a través de ocupaciones y asentamientos informales).

La pérdida de población en los barrios tradicionales y consolidados de la ciudad ${ }^{13}$ se produce simultáneamente con la migración hacia la periferia y la concentración de problemas sociales en determinadas áreas. Se identifican dos dinámicas particularmente visibles asociadas al modelo de crecimiento por extensión: a) la intensificación de la segregación residencial y b) la fragmentación física en el patrón de ocupación del espacio con un crecimiento insular, donde la trama abierta de manzanas y calles es reemplazada por tramas cerradas y discontinuas. Este patrón está presente tanto en los desarrollos del mercado inmobiliario para sectores de altos ingresos (con límites físicos en el perímetro y accesos controlados) como en los barrios destinados a población vulnerable, materializados a través de políticas públicas de vivienda, que por la escala de intervención y las

12 Abramo, 2003.

13 Marengo, 2008. condiciones de aislamiento en la localización, se configuran como fragmentos diferenciados en la estructura urbanizada.

Estudios sobre segregación en ciudades norteamericanas $^{14}$ evidencian que aquellas áreas con altos niveles de segregación social (específicamente cuando la concentración de población pobre alcanza el 30\%) evolucionan en su desarrollo, intensificando esta condición. Como resultado, la situación urbana en dichas áreas no es sustentable en términos económicos (localización de inversiones, acceso a condiciones de trabajo, valorización de las propiedades) ni sociales (mayores niveles de violencia urbana y conflictos).

\section{ABORDAJES Y DIMENSIONES ANALÍTICAS DE LA SEGREGACIÓN RESIDENCIAL}

En muchas oportunidades se hace referencia de manera indistinta a los procesos de división social del espacio y a la segregación residencial. Duhau establece una diferenciación entre ambos. En el primer caso se refiere a las diferencias existentes en la localización intra-urbana o intra-metropolitana de diferentes grupos o clases sociales, relacionadas fundamentalmente con las posibilidades de acceso al suelo en el mercado. En el segundo, entiende la segregación residencial como un proceso

14 Rusk, 1999. 
de división social del espacio promovido a través de medidas coercitivas, producto de la exclusión forzada o explícitamente buscada en relación con otros grupos sociales determinados (ya sea mediante políticas o prácticas, como por ejemplo el apartheid en Sudáfrica) ${ }^{15}$. Es decir, la segregación está relacionada con la localización residencial, ya sea por libre elección o debido a la implementación de determinadas políticas de acceso al suelo o a la vivienda.

En este trabajo adherimos a la conceptualización de la segregación como "una relación espacial que atañe a la proximidad territorial entre personas pertenecientes a un mismo grupo socioeconómico"16. Entendemos la segregación como el grado de mixtura presente en la estructura urbana (niveles de homogeneidad o heterogeneidad) y la distancia geográfica entre grupos sociales en su distribución en la ciudad. La segregación sería inexistente si las diferentes categorías socioeconómicas consideradas en el análisis de la distribución de la población en la ciudad, estuvieran mixturadas en todas las escalas territoriales de análisis ${ }^{17}$.

La segregación residencial presenta tres dimensiones analíticas: i) el grado de concentración espacial de los grupos sociales (en términos de localización urbana), lo que implica un análisis relativo de la

15 Duhau, 2013.

16 Sabatini, 2004, p. 279

17 Clichevsky, 2000, p. 9. posición / localización de un determinado grupo en relación al conjunto; ii) la homogeneidad en las diferentes áreas de la ciudad, en términos de su composición social (lo que implica focalizar el análisis en la dimensión territorial-urbana; las capacidades urbanas instaladas y las ventajas relativas que ciertos sectores presentan dentro de la ciudad, por ejemplo en términos de dotación de servicios, acceso a la infraestructura y a la movilidad, condiciones de accesibilidad, entre otras) y iii) la percepción que las personas tienen de la segregación como fenómeno socio-espacial ${ }^{18}$.

Las dos primeras dimensiones hacen referencia a aspectos objetivos de la condición socio-espacial, mientras la última remite a aspectos subjetivos. Se relaciona con la identidad y con el prestigio asignado a determinados barrios o zonas de la ciudad en un devenir histórico (barrios obreros, barrios de estudiantes, zonas comerciales o turísticas, áreas peligrosas, etc.). La percepción social de los atributos de una determinada área contribuye a la construcción de estigmas territoriales, con efectos en la integración social de los residentes y en las posibilidades de acceso a las oportunidades derivadas de la aglomeración.

Rodrígue $z^{19}$ refiere que en las dos primeras dimensiones (objetivas) hay una cierta superposición, ya

\footnotetext{
18 Sabatini, 2004.

19 Rodríguez, 2001.
} 
que la localización de un grupo en una cierta área implica la configuración de un sector homogéneo, sin embargo, la distinción de estas dos dimensiones tiene sentido. La primera dimensión tiene lugar cuando algún grupo social registra un sesgo residencial global, es decir, cuando todos (o la gran mayoría) sus miembros se localizan en una zona determinada del territorio, sin importar si en dicha zona hay otros grupos sociales. Esta segregación por localización de grupo opera cuando, en una situación en la que hay varios grupos sociales, uno o más no están dispersos por el territorio sino que se encuentran concentrados en una zona específica. En cambio, la segunda dimensión, que puede denominarse por exclusión, atañe a la ausencia de mezcla o integración de grupos sociales en espacios comunes. En esta dimensión, un grupo social no se mezcla con el resto aunque esté diseminado en varias partes de la ciudad; situación que se ve reforzada en el último período con la materialización de muros que delimitan determinados territorios. Así se originan zonas homogéneas en un contexto heterogéneo, lo que probablemente dificulta la interacción con otros grupos sociales.

Por otra parte, Saravi ${ }^{20}$ enriquece el análisis aclarando que cuando hablamos de la segregación en términos de la distribución de la población en el espacio, del nivel de concentración de determinados

20 Saravi, 2008. grupos en ciertas áreas de la ciudad y/o del grado de homogeneidad social que presentan áreas específicas, no se trata simplemente de un criterio de diferenciación, sino al mismo tiempo de un criterio clave de jerarquización y más específicamente de desigualdad social.

A partir de esta perspectiva se comprende al fenómeno desde sus dimensiones espacial y social. La conformación de los territorios para los sujetos es una práctica simbólica, al otorgarle distintos significados al territorio en el que habitan (arraigo, pertenencia, identidad, etc.) y a su vez, las representaciones sociales construidas en torno a la diferenciación territorial también se constituyen en fuentes de desventajas e instrumentos de sostenimiento de las desigualdades sociales.

Las modalidades diferenciadas en que los grupos poblacionales acceden a la ciudad y a los recursos urbanos, ponen en evidencia procesos de desigualdad social que tienen su correlato en lo territorial $^{21}$. Como plantea Cuenya ${ }^{22}$, los estudios sobre segregación residencial hablan del surgimiento de guetos urbanos (que hasta hace poco eran propios de las ciudades americanas) para describir una realidad de profundización de la pobreza, en términos de aumento de la vulnerabilidad de los pobres por: pérdida de oportunidades laborales, educativas y recreativas; mayor exposición a la

21 Di Virgilio y Perelman, 2014.

22 Cuenya, 2014,. p. 97. 
violencia e inseguridad; disminución de la capacidad de acumulación de recursos, y fragilidad de las relaciones con personas de distinta condición socioeconómica, entre otros factores.

\section{POLÍTICAS DE VIVIENDA SOCIAL Y LOCALIZACIÓN URBANA}

Históricamente, las políticas de vivienda social han sido criticadas por la localización periférica de los proyectos habitacionales, con limitaciones en el acceso a los servicios públicos y en equipamientos sociales y culturales ${ }^{23}$. Sin embargo en las últimas décadas, con los procesos de creciente mercantilización del desarrollo urbano donde la plusvalía urbana ha pasado a ser el criterio urbanístico dominante ${ }^{24}$, las condiciones de localización de los nuevos proyectos habitacionales lejos de mejorarse han empeorado y el déficit en la accesibilidad a los recursos y ventajas de la aglomeración se ha intensificado. Esta fue una de las consecuencias derivadas de las redefiniciones ideológicas que atravesó la política de vivienda en América Latina y el mundo, en el contexto del predominio del capital financiero global y del aumento del rol de las agencias multilaterales de crédito $^{25}$. Los nuevos lineamientos se basaron en los principios de "facilitación y focalización", los que determinaron

23 Yujnovski, 1984; Pradilla Cobos, 1987.

24 Mattos, 2010.

25 Fernández Wagner, 2014. el rol de los actores y agentes estatales, privados y comunitarios en la satisfacción de las necesidades de hábitat. Para ello, el Estado debía estructurar ese marco cumpliendo un rol de facilitador de las iniciativas particulares, es decir, dejar de lado su anterior función de productor de vivienda y asumir el papel de administrador en la totalidad del sector y de orientador del desempeño del ámbito privado estructurado y no estructurado, poniendo más énfasis en la función global que cumple la vivienda en el desarrollo económico y social ${ }^{26}$.

Numerosos estudios muestran los resultados de la política de vivienda efectuada bajo este enfoque. Chile es uno de los países de la región donde ha habido un importante desarrollo académicocientífico en los últimos años respecto a este tema; distintos autores analizan la estrecha relación entre las políticas de vivienda social y la producción de la segregación residencial. Hidalgo ${ }^{27}$ plantea que lo novedoso de la política de vivienda de país es que los nuevos proyectos habitacionales ya no se encuentran en la periferia de la ciudad motorizando la expansión urbana, sino que se levantan en espacios distantes, tanto en el medio rural como en las ciudades menores existentes en los confines de las grandes conurbaciones, profundizando los

26 Ibíd.

27 Hidalgo, 2007. 
procesos de segregación social y fragmentación física del espacio urbano ${ }^{28}$.

En México, la política de vivienda social producida desde los años 90 se ha caracterizado por la activa participación del sector inmobiliario, con intervención no solo en la construcción de viviendas sino también en la adquisición del suelo, urbanización, promoción e incluso en los mecanismos de financiamiento, instaurándose un sistema predominantemente privatizado y mercantilizado ${ }^{29}$. Esto ha tenido como correlato la construcción de megaproyectos residenciales de gran superficie y elevado número de viviendas, emplazados en municipios distantes de las grandes ciudades del país, sin una adecuada previsión para la dotación de servicios urbanos y comunitarios ${ }^{30}$.

Las políticas de vivienda de Chile y México fueron referencias importantes para la formulación en Brasil del programa "Mi Casa, Mi Vida", que también estuvo basado en la producción de nuevas unidades por producción privada, con proyectos habitacionales localizados en los bordes de las ciudades, en áreas carentes de empleo, infraestructura y equipamientos, desarticuladas de los contextos urbanos, lo que crea una demanda y un costo financiero y personal adicional de transporte ${ }^{31}$.

Rodríguez y Sugranyes, 2005; Brain y Sabatini, 2006.

Esquivel, Maya y Cervantes, 2005.

Rodríguez, 2006.

Bonduki, 2014.
En Argentina, el informe del Programa de Naciones Unidas para el Desarrollo ${ }^{32}$ (PNUD) analiza el fenómeno de la segregación residencial socioeconómica y plantea las limitaciones de las políticas implementadas en los últimos quince años dirigidas a abordar la informalidad urbana en las principales ciudades del país ${ }^{33}$. Se constata que los distintos programas (de regularización urbana y dominial de villas y asentamientos, así como los programas de relocalización) no aportan mixtura social y localizan población con alto grado de vulnerabilidad en barrios históricamente pobres, lo cual trae aparejado la reproducción de las condiciones de pobreza.

\section{Metodología para la medición de la segregación residencial socioeconómica}

Como señalamos anteriormente, en este trabajo abordamos la dinámica de la segregación residencial socioeconómica en la ciudad en tres cortes temporales, 1991, 2001 y 2008 en especial, y los niveles de homogeneidad / heterogeneidad de distintos sectores urbanos.

\footnotetext{
32 PNUD, 2009

33 Este estudio se realizó en el Área Metropolitana de Buenos Aires (AMBA), Córdoba, Rosario y Mendoza.
} 
En la última década del siglo XX, se inicia en Córdoba el desarrollo de grandes emprendimientos comerciales y residenciales promovidos por la inversión privada y simultáneamente acciones de política urbana, orientadas a la descentralización administrativa del municipio y a la provisión de servicios en la periferia urbana. Estas acciones modifican la estructura tradicional de ciudad monocéntrica, evidenciando una tendencia hacia una estructura urbana policéntrica. En la primera década del siglo XXI se intensifica la extensión física con el desarrollo de urbanizaciones cerradas para altos ingresos en la periferia y el área metropolitana y simultáneamente se desarrolla una política de construcción de vivienda social de gran escala para relocalizar a sectores vulnerables que residían en áreas con riesgo de inundación. Estas acciones impactan en la estructura socio-territorial y en la segregación residencial.

La metodología aplicada es cuantitativa; se realizó la medición de la SRS en los tres cortes intercensales (1991, 2001 y 2008) que permiten identificar los cambios en la dinámica del fenómeno y aportan un análisis comparativo. El estudio, a escala de fracciones censales, posibilitó detectar las áreas urbanas que presentan alta homogeneidad respecto de los grupos que las habitan. Debido a que los censos nacionales de población y vivienda en Argentina no incorporan indicadores de medición de la segregación, se tomó como variable de segmentación socioeconómica los años de estudio del jefe del hogar, lo que permite extrapolar la pertenencia social de los hogares en diferentes grupos socioeconómicos ${ }^{34}$.

Si bien existen varios métodos de medición de la segregación ${ }^{35}$, el análisis del nivel de homogeneidad-heterogeneidad en las distintas subdivisiones territoriales (fracciones censales) se realizó a partir de la media de la variable seleccionada y del cálculo estadístico de la varianza y del coeficiente de variación, que expresa la magnitud de la desviación estándar en función de la media de la distribución ${ }^{36}$. Para dicho cálculo se utilizaron datos secundarios: el Censo de Población Nacional del

34 Duhau (2013) señala que para estratificar a los individuos u hogares, la mayor parte de los investigadores optan por recurrir a variables tales como ingreso, escolaridad, ocupación o bienes poseídos. La elección de la variable que establecemos se basa en el supuesto de que existe una correlación entre la educación del jefe de hogar y la probabilidad de obtener mayores ingresos familiares.

35 Sobre la discusión de los distintos métodos se puede remitir a Arriagada y Rodríguez, 2003; Sabatini, 2004.

36 Arriagada, Luco y Rodríguez, 2003. 
año 1991, 2001, realizado por el Instituto Nacional de Estadísticas y Censos (Indec), y el Censo Provincial de Población 2008, dado que a la fecha de desarrollo de la investigación no se contaba aún con los datos procesados del Censo Nacional de Población y Vivienda 2010.

El procedimiento de medición de la segregación se dividió en dos instancias: primero, se calculó la media de años de estudios de los jefes de hogar para toda la ciudad y el valor del coeficiente de variación de cada unidad geográfica (fracciones censales) respecto de aquella para los tres cortes temporales (1991, 2001 y 2008). Con los resultados obtenidos se realizó una clasificación en cuatro intervalos, según la intensidad del coeficiente de variación de la variable analizada por fracción. Es decir, se consideró, en un primer intervalo, aquellos valores de variación comprendidos entre el $1 \%$ y el $10 \%$ (por encima y por debajo de la media), lo cual señala heterogeneidad en la distribución y, consecuentemente, un bajo valor de segregación. El segundo intervalo agrupa los valores comprendidos entre el $11 \%$ y el $20 \%$ (por encima y por debajo de la media ${ }^{37}$ ), los cuales se consideran valores moderados de segregación. El tercer intervalo incluye valores comprendidos entre el $21 \%$ y el 30\% (por encima y por debajo de la media), que

37 Los valores por encima de la media se asocian con sectores sociales de ingresos medio altos/muy altos, y los valores por debajo de la media con sectores sociales de ingresos medio bajos/ muy bajos. se consideran valores altos de segregación. Finalmente, el cuarto intervalo abarca valores que superan el 30\% (por encima y por debajo de la media) que se consideran de muy alta segregación. Estos intervalos han sido representados en planos de la ciudad (gráficos 1, 2 y 3) a los efectos de identificar los sectores urbanos en situación de mayor homogeneidad - heterogeneidad en los diferentes cortes temporales y analizar su evolución y la vinculación con localización de la vivienda pública en el territorio.

\section{La evolución de la segregación residencial socioeconómica en Córdoba en el período 1991-2008}

La ciudad de Córdoba desde sus orígenes se ha caracterizado por ser segregada y su producción se ha configurado desde una lógica de diferenciación en la localización residencial de los distintos grupos sociales. La mayoría de la población de bajos ingresos sigue habitando en las áreas periféricas ${ }^{38}$, históricamente conformadas con menor oferta urbana, lo cual se traduce en menores oportunidades

38 Marengo, 2004; Tecco y Fernández, 2005; Marengo y Elorza, 2014; Molinatti, 2013. 
para el acceso a servicios y equipamientos sociales (educativos, culturales, recreativos, etc.).

Los resultados del análisis efectuado para el periodo 1991-2008 indican que en Córdoba se mantiene el patrón tradicional de segregación de las ciudades de América Latina, ya que los sectores de altos ingresos se localizan en el área central (proceso que se ha profundizado en 2008) y se expanden hacia el noroeste conformando un "cono de alta renta"39; en el área intermedia de la ciudad hay una mayor presencia de sectores medios; y en el área periférica (con excepción del eje noroeste) predomina la población de bajos ingresos.

Sin embargo, se han producido modificaciones en el territorio que es necesario analizar:

Periodo 1991-2001: durante estos años la segregación residencial se intensificó en las áreas periféricas de la ciudad, aumentando la distancia geográfica entre los grupos sociales de altos ingresos localizados en el sector noroeste de la ciudad y los grupos de bajos ingresos localizados en el sureste. Numerosas áreas urbanas perdieron heterogeneidad socioeconómica y la mixtura social que tenían a comienzos de la década de los noventa; en especial, se observa un aumento de la concentración de la población de bajos ingresos en determinadas

39 Sabatini, 2004. áreas del territorio, asociado a las acciones estatales en materia habitacional promovidas por el estado local o provincial. La manera de afrontar la problemática de la informalidad urbana (con acciones de relocalización de población pobre en los bordes urbanos) y el tipo de programas habitacionales (vivienda "llave en mano") han contribuido a consolidar áreas monofuncionales, sin mixtura social, reproduciendo un mismo patrón de ocupación físico-espacial.

Periodo 2001-2008: la dinámica de este fenómeno muestra un comportamiento contradictorio. Por un lado, se han ampliado las áreas de baja y moderada segregación, lo que significa una mayor dispersión en la localización de los sectores de ingresos medios en la ciudad; a su vez, el proceso de localización de los grupos de bajos ingresos ha tenido una dinámica de dispersión en el sector periférico de la ciudad. Por otro lado, en sentido opuesto, se han consolidado los sectores de la ciudad identificados con alta y muy alta segregación (población de más alto ingreso), concentrándose en el área central y noroeste de la ciudad.

Si bien durante estos años han mejorado las condiciones de vida de la población de bajos ingre$\operatorname{sos}^{40}$, el valor del suelo ha aumentado de manera

40 En la ciudad de Córdoba ha disminuido la población bajo la línea de pobreza desde $24,7 \%$ en 2001 al 14,8\% en 2008; y también se registró una caída en la población en condiciones de indigencia, desde $7 \%$ al $2,6 \%$ en el mismo periodo. 
exponencial, haciéndose aún más difícil el acceso a un terreno urbanizado para las familias de ingresos medios y bajos, condición que también afecta la localización de proyectos habitacionales desarrollados a través de políticas de vivienda social ${ }^{41}$.

Se identifica que la forma de crecimiento que prevalece en este período (extensión dispersa) reproduce condiciones de alta inequidad espacial. Las zonas intermedias de la ciudad que cuentan con provisión de infraestructura, servicios urbanos y mayor heterogeneidad social pierden población, mientras que en los bordes urbanos se incrementa la cantidad de población. La relación entre expansión urbana y segregación residencial evidencia el aumento de la distancia espacial y social entre los diferentes grupos poblacionales y la consolidación de áreas socialmente homogéneas en los bordes de la ciudad. En este sentido, en el último corte temporal analizado (2001-2008) se intensifica la "segregación por localización de grupo"42, con sectores de altos ingresos concentrados en una zona específica de la ciudad: el área central y la zona noroeste.

Como plantean Brain y Sabatini, la segregación de los grupos de menores ingresos en la periferia tiene impactos urbanos y sociales. Entre los primeros destacan los problemas de accesibilidad y la carencia de servicios y equipamientos de cierta calidad en sus lugares de residencia; y entre los segundos, los problemas de desintegración social que están escalando hoy y representan formas de empobrecimiento o de degradación social vinculadas a las desventajas que conlleva el aislamiento físico, lo que hace que los grupos populares de nuestras ciudades sean aún más pobres ${ }^{43}$.

La desigual apropiación de la ciudad por los distintos grupos sociales, marcadamente diferenciada en tanto oportunidades y acceso a bienes y servicios, no la podemos analizar sin tener en cuenta la lógica del mercado de suelo. Si consideramos el suelo urbano como una mercancía con valor de cambio, los sectores de bajos ingresos solo pueden acceder a áreas donde los valores de la tierra urbana son bajos o muy bajos. Esta lógica también permite a los sectores de altos ingresos desarrollar estrategias de distinción social, apropiándose de las áreas urbanas de mejor calidad (con accesibilidad, servicios y cualidades ambientales), garantizando la exclusión de grupos poblacionales de otra condición social.

\footnotetext{
43 Brain y Sabatini, 2006.
}
41 Un estudio de Monayar (2014) revela cómo se incrementa el pre- cio medio de un terreno de 300 m2 en Córdoba: en 1990 era de USD \$3.946, en 2001 de USD \$13.371 y en 2008 de USD \$40.051 (p. 158).
42 Rodríguez, 2001.


GRÁFICO 1: SRS EN CÓRDOBA EN 1991.

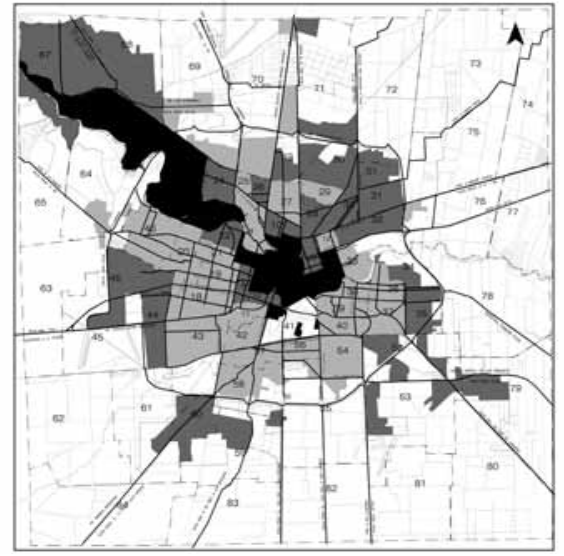

GRÁFICO

Segregación socioeconómica según nivel de estudios de hogar (1991).

Variación del 1 al 10\%

Variación del 11 al 20\%

- Variación del 21 al $30 \%$

Variación superior al $30 \%$

Fuente: Censo Nacional de Población y Vivienda 1991.

Elaboración propia.

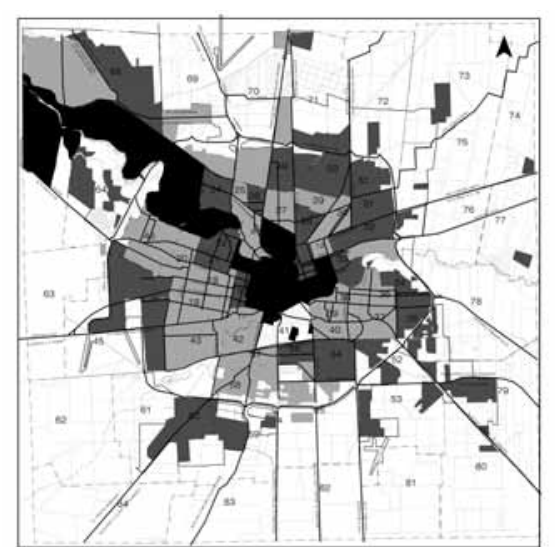

GRÁFICO 2: SRS EN CÓRDOBA EN 2001.

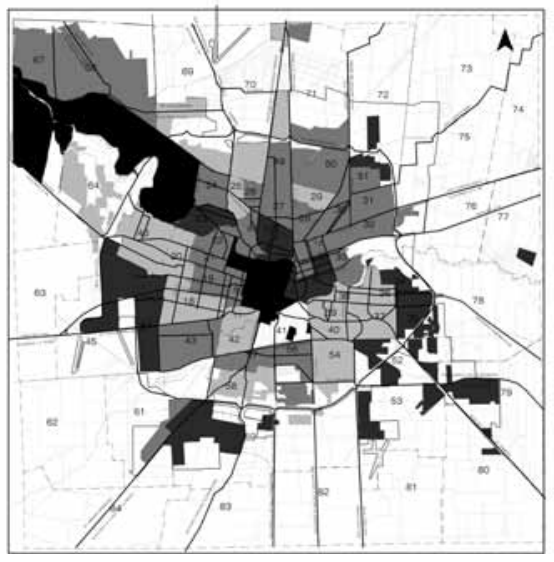

GRÁFICO

Segregación residencial socioeconómica según nivel de estudios del jefe de hogar (2001).

Variación del 1 al $10 \%$

Variación del 11 al 20\%

Variación del 21 al $30 \%$

Variación superior al $30 \%$

Fuente: Censo Nacional de Población y Vivienda 2001.

Elaboración propia.

GRÁFICO 3: SRS EN CÓRDOBA EN 2008.

\section{GRÁFICO}

Segregación residencial socioeconómica según nivel de estudios del jefe de hogar (2008)

Variación del 1 al 10\%

Variación del 11 al 20\%

Variación del 21 al $30 \%$

Variación superior al 30\%

Fuente: Censo Nacional de Población y Vivienda 2008.

Elaboración propia

ARTíCULO: Vivienda social en Córdoba, efectos en la segregación residencial y el crecimiento 


\section{LOS PROGRAMAS DE VIVIENDA SOCIAL EN CÓRDOBA (1991-2008)}

La década del 90 impulsó cambios significativos en las políticas urbanas que se reflejaron en un crecimiento económico. En el marco de los procesos de internacionalización de la economía, el gobierno local promovió el desarrollo de programas de planificación estratégica y favoreció la participación ciudadana y las acciones de los agentes económicos para atraer nuevas inversiones y hacer más competitiva la ciudad. Estas condiciones se mantuvieron hasta 1997, cuando comenzó la recesión económica que hace su pico en diciembre de 2001 con el default de Argentina.

A partir de 1991, las políticas de vivienda se orientaron a la descentralización de los recursos, la diversificación de los programas, la creciente preocupación por mejorar la eficiencia y la eficacia de las acciones y promovieron la incorporación de actores sociales. En el marco del Fondo Nacional de Vivienda (FONAVI), la Secretaría de Estado de Desarrollo Urbano y Vivienda es la responsable de los aspectos generales de planificación, determinación de prioridades y normas de las operatorias respectivas, siendo responsabilidad de los Institutos Provinciales de Vivienda (IPV), la ejecución de los programas: contratación y control de las obras, registro de solicitantes y adjudicación. En el año
1992 se transfieren los recursos del FONAVI a los IPV a través de la ley 24.130. "El 'acuerdo interestadual' suscrito entre el gobierno nacional y los gobiernos provinciales sobre el uso y administración de fondos coparticipables y fondos especiales otorga a los entes ejecutores de cada jurisdicción provincial la responsabilidad exclusiva del otorgamiento de aptitudes técnicas y financieras de cada proyecto habitacional" ${ }^{\prime 44}$.

A partir de esta modificación en la estructura organizativa, política y financiera, en Córdoba se crea el Fondo de Vivienda de Córdoba (FOVICOR). Este fondo contaba con: los aportes del presupuesto general de la provincia, el recupero de los créditos ya otorgados y los recursos provenientes del FONAVI. A pesar de contar con la capacidad de definición de los destinatarios, el Instituto Provincial de la Vivienda mantuvo los criterios de selección: sectores de ingresos medios y bajos, capaces de afrontar una cuota mensual para el pago de la unidad habitacional.

Durante el periodo 1991-1999 se desarrollaron distintas estrategias para abordar la problemática habitacional de la población con alto grado de vulnerabilidad: programas de relocalización, proyectos de radicación in situ y programas de gestión asociada entre el Estado y las organizaciones sociales. En el primer caso se erradicaron villas

44 Cuenya, 1997. p. 15. 
localizadas en terrenos fiscales para realizar obras públicas (red vial de la costanera del río Suquía y del arroyo La Cañada y nudos viales), incluyeron el traslado de 501 familias. La segunda estrategia, de radicación in situ, significó un cambio en la forma de trabajo y en las soluciones habitacionales destinadas a la población vulnerable, se logró regularizar la situación de informalidad de aproximadamente 490 familias.

La tercera estrategia, de gestión asociada, se implementó a través de la Mesa de Concertación de Políticas Sociales, en la cual participaban el Estado Provincial y Municipal, ONG's y la Unión de Organizaciones de Base por los Derechos Sociales (UOBDS). El objetivo de la misma era discutir las políticas y herramientas encaminadas a mejorar la calidad de vida de la población vulnerable. Como alternativa a las prácticas tradicionales, se convocó a los beneficiarios (a través de sus organizaciones sociales y con el asesoramiento de equipos técnicos) a presentar al gobierno sus demandas para el mejoramiento de sus condiciones de vida. En el marco de esta experiencia, se solicitó al municipio la creación de un banco de tierras, la necesidad de un tratamiento impositivo diferenciado en el caso de la vivienda social y la creación de un mecanismo más ágil para la aprobación de urbanizaciones.
El gobierno local intervino asumiendo la provisión de infraestructura básica en los programas habitacionales concertados, ampliando la cobertura de los beneficiarios de los programas. La participación de los destinatarios en el proceso de planificación contribuyó a la organización e inserción de la comunidad realojada. Se formularon instrumentos normativos que flexibilizaron las exigencias de urbanización, como por ejemplo: la reducción de las dimensiones mínimas de las parcelas exigidas para viviendas individuales (lotes de $160 \mathrm{~m} 2$ de superficie mínima), el requerimiento de proveer infraestructura básica para los loteos sociales y la posibilidad de ejecutar la infraestructura requerida por etapas.

Si bien hacia el final del período se efectuaron algunos programas piloto de radicación in situ ${ }^{45}$, o de realojamiento de población manteniendo la proximidad con la ubicación inicial, no se observan cambios significativos en la localización urbana de los conjuntos (periférica) o en el tipo de operatorias (que continúan centradas en la vivienda "llave en mano"). Esta política habitacional se desarrolló hasta 1995 y con el cambio de gobierno se retomó la política de erradicación de población villera del área central e intermedia de la ciudad hacia la periferia urbana (gráfico 4).

45 Por ejemplo, el reasentamiento de la villa de emergencia 4 de agosto, en el barrio Marqués de Sobremonte, que incluyó 400 familias. 


\section{GRÁFICO 4: LOCALIZACIÓN DE VIVIENDA SOCIAL EN CÓRDOBA 1991-2008.}

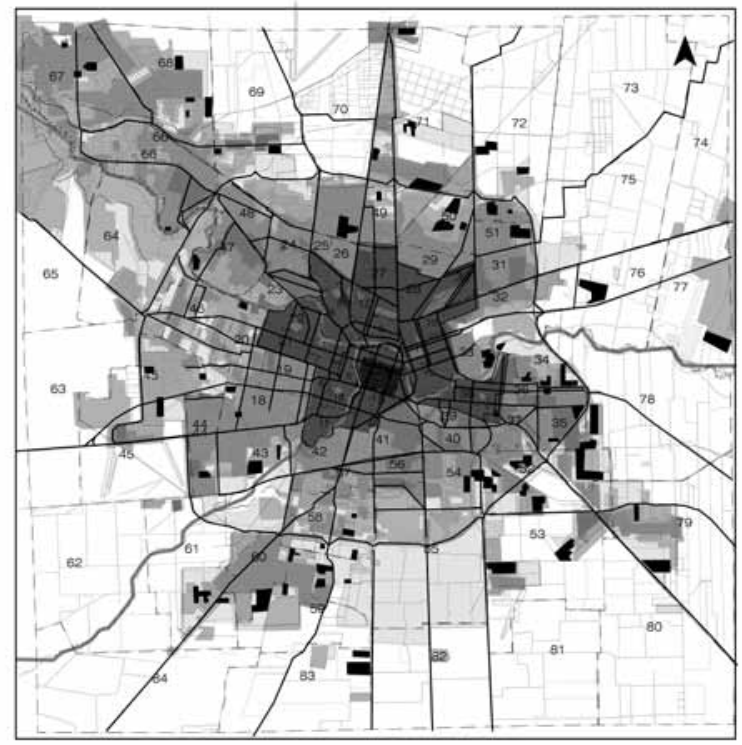

GRÁFICO

Localización de vivienda social 1991 - 2008.

Vivienda social

Fuente: Ministerio de Desarrollo Social - Gobierno de la Pvcia. de Córdoba Elaboración propia.

En el año 2001, el poder ejecutivo de la Provincia de Córdoba declara la emergencia hídrica y la urgente relocalización de los grupos vulnerables afectados por las inundaciones. Dicho plan se difundió públicamente como el programa "Mi Casa, Mi Vida" o el "plan de las 12.000 viviendas", que era la meta a alcanzar en la producción de unidades habitacionales. El desarrollo del programa comprendió el periodo 2003-2010 y su financiamiento se solventó a través de un crédito otorgado por el Banco Interamericano de Desarrollo (BID), que abarcó el 83\% del presupuesto. Como contraparte se utilizaron fondos del presupuesto provincial; el costo fue de US\$ $116.036 .200^{46}$.

El objetivo general fue contribuir al mejoramiento integral del hábitat y las condiciones de vida de los grupos vulnerables que habitaban zonas de riesgo y fueron afectados por las inundaciones. Con un fuerte criterio de focalización, la población objetivo de este programa fueron los residentes de villas de emergencia, incapaces de acceder a una vivienda en el mercado formal y en condiciones críticas de habitabilidad. Los barrios, con una cantidad mayor a 250 unidades, contaron con equipamiento a escala de sector urbano (escuelas, centro de salud, posta policial, comedor infantil, comercios, etc.). Fueron denominados barrios-ciudades por la magnitud de las intervenciones (por ejemplo, Ciudad Evita, Ciudad de Mis Sueños, etc.). Atendiendo a la integralidad de la problemática habitacional, el programa se configuró a través de cuatro

46 El costo de cada solución habitacional no debía superar los USD $\$ 8.600$ con infraestructura de servicios básicos, equipamiento social mínimo y terreno. El programa preveía la entrega de la vivienda completamente subsidiada. 
componentes: urbano, legal, social y ambiental. El componente urbano incluyó la construcción de unidades de $42 \mathrm{~m} 2$ con baño, cocina comedor y 2 dormitorios en terrenos individuales, no mayores a 300 m2 y provisión de los servicios de agua, electricidad, cloacas, e infraestructura: asfalto, alumbrado público, espacios verdes, etc. El componente legal incluyó el acompañamiento social a las familias durante el proceso de traslado, la promoción de la organización vecinal, el trabajo en red con las instituciones del barrio (escuela, dispensario, comedor de ancianos, etc.), actividades de desarrollo laboral, promoción infantil y adolescente y titularidad de la vivienda. El componente ambiental preveía la recuperación de las zonas desalojadas.

Si bien este programa no tuvo como objetivo abordar el fenómeno de la segregación, su acción generó efectos en la configuración de la ciudad y en la localización residencial de población de bajos ingresos, lo cual podemos comprenderla, en términos de Bourdieu ${ }^{47}$, como una construcción política del espacio. Durante el periodo 2003-2008 se trasladaron aproximadamente 70 villas de emergencia y se adjudicaron 8.876 viviendas emplazadas en 39 nuevos barrios. Se ejecutaron nueve barriosciudades con más de 250 viviendas cada uno. En el año 2001, la cantidad de hogares con déficit habitacional cuantitativo en Córdoba era de 20.311 y

47 Bourdieu, 2001. la implementación de este programa dio respuesta al 43,7\% del mismo.

El acceso a la vivienda y a los servicios públicos ha representado mejores condiciones de vida para muchas familias; sin embargo, no podemos decir que los problemas habitacionales se hayan resuelto. Desde la lógica "viviendista" como plantea Rodulfo ${ }^{48}$, los nuevos barrios de vivienda social son concebidos como una ciudad dentro de la ciudad. El proyecto responde al imperativo de eficiencia en el polígono de intervención y el diseño incorpora una organización física diferenciada. Esta lógica se expresa en la ejecución de proyectos idénticos (tipologías, diseño de espacios verdes y de equipamiento, etc.) en diferentes sectores urbanos (algunos desvinculados del entorno edificado) con una respuesta edilicia homogénea que no reconoce rasgos culturales y de identidad, necesidades habitacionales según el tipo de hogares, así como condiciones de localización que consideren la inserción previa de la población trasladada.

En cuanto a las dimensiones y tipología de la vivienda, ésta ha sido diseñada desde parámetros del modelo de familia nuclear, obligando a otros tipos de hogares (nuclear-numerosa, extensos, etc.) a acomodarse en reducidos espacios, con umbrales críticos que pueden tener como consecuencias problemas psicológicos, afectivos y de convivencia.

48 Rodulfo, 2006. 


\section{GRÁFICO 5: LOCALIZACIÓN DE LA VIVIENDA SOCIAL EN LAS FRACCIONES 53 Y 79.}
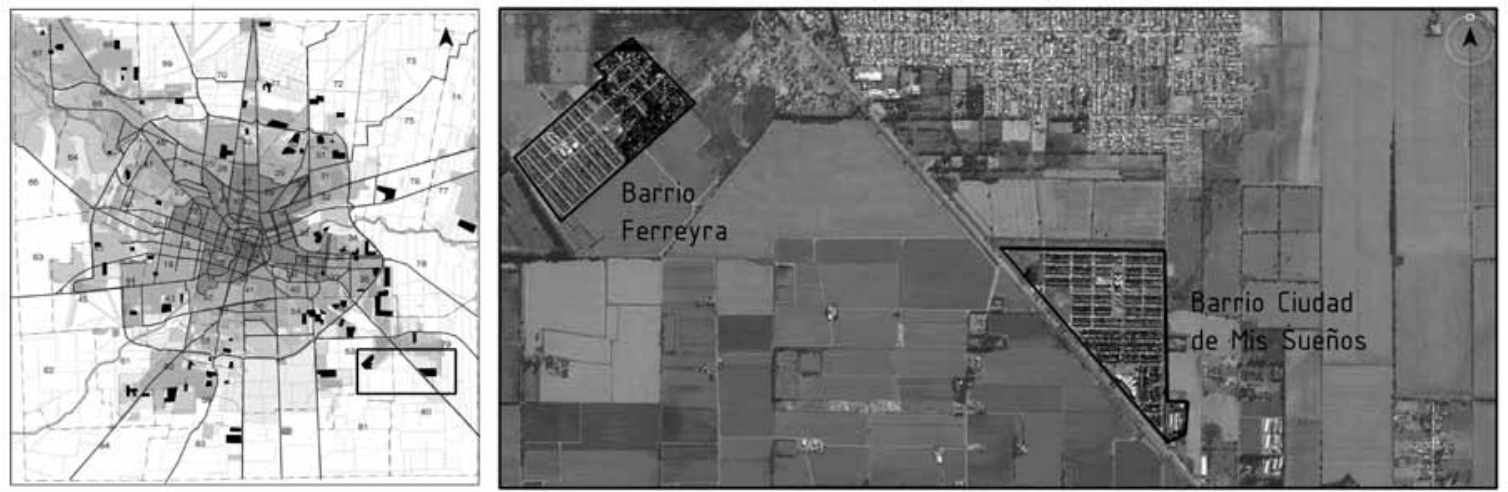

GRÁFICO

VIVIENDA SOCIAL - RECORTE DE LAS FRACCIONES 53 Y 79.

Fuente: Google Earth 2008.
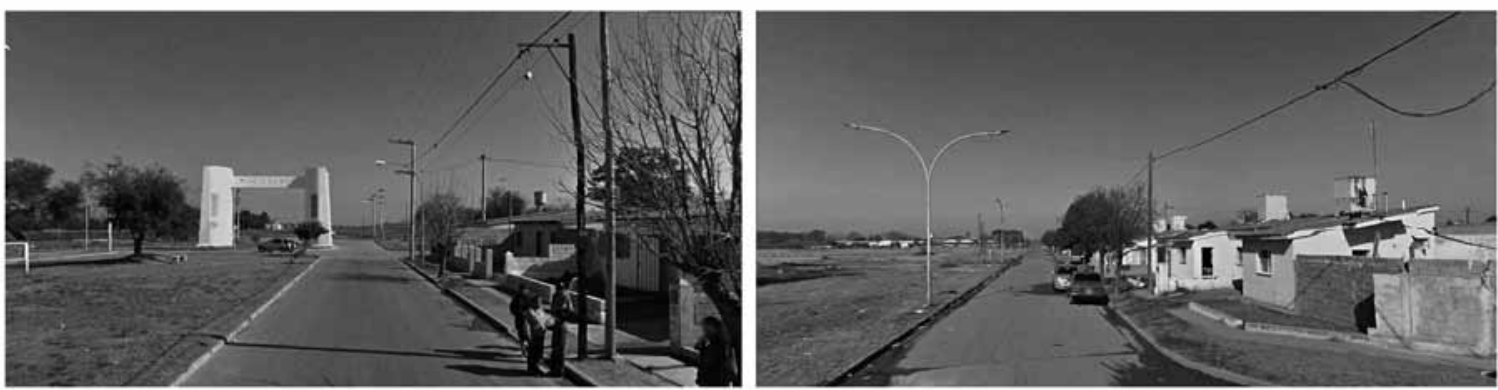

Fotografías de la Ciudad de Mis Sueños.

Fuente: Google Street View 2008.

De esta manera, aun cuando el programa ha tenido gran impacto en términos del acceso a la vivienda, siguen reproduciéndose condiciones habitacionales deficitarias ${ }^{49}$.

49 Elorza, 2008. 
La localización de los conjuntos habitacionales ha configurado una nueva distribución de la población pobre en la ciudad, trasladándola desde el anillo central e intermedio a sectores periféricos, donde los servicios y equipamientos son de menor calidad. Los nuevos barrios se localizan en el borde atendiendo al menor valor del suelo, generalmente incorporando suelos afectados al uso rural, con la consecuente demanda de extensión de infraestructura y servicios, con implicancias en los costos de urbanización y de funcionamiento que deben ser asumidos por el municipio (gráfico 5).

\section{Conclusiones}

En los últimos años, estudios realizados sobre la implementación de políticas de vivienda social en distintas ciudades de América Latina dan cuenta de una constante localización de los proyectos habitacionales en los bordes urbanos, condición que también se verifica en el caso de estudio, atendiendo a ofertas de menor valor en las parcelas para ser urbanizadas.

Hacia el final del período analizado, esta condición se agudiza al combinar criterios de focalización de la demanda social con operaciones de gran escala de intervención. El caso de Córdoba evidencia que la política habitacional incide negativamente en la extensión del crecimiento físico, promueve un patrón de ocupación difuso e insular cada vez más periférico e intensifica la segregación residencial y la distancia física entre la localización de población de altos y bajos ingresos en la estructura urbana.

El mapeo de las condiciones de segregación residencial (a escala de fracciones censales) y el estudio de su evolución en el tiempo, aportan un diagnóstico que alerta sobre situaciones críticas presentes en determinadas áreas del borde urbano. También ofrece una herramienta de planificación que debe ser tenida en cuenta al momento de evaluar la futura localización de planes de vivienda social y su diseño, en términos de escala (cantidad de unidades), mixtura social (perfil de la demanda) y diversidad edilicia (diseño de las unidades habitacionales), entre otros.

Consideramos que la planificación de la vivienda social debe estar inserta en una política de desarrollo urbano, porque es una herramienta que puede atenuar condiciones de segregación residencial, dinamizar áreas deprimidas y contribuir a integrar a la población pobre en la ciudad. Sin embargo, el caso analizado pone en evidencia una situación paradójica. Aun cuando han mejorado las condiciones de pobreza estructural en el país y se ha desarrollado una política habitacional de gran impacto en términos de construcción de unidades habitacionales, la segregación residencial en el territorio urbano se ha intensificado, lo que significa que existen verdaderas áreas-problema, en términos de integración urbana. 
La prevalencia de la lógica del mercado en la producción del hábitat social relega las condiciones de localización a la oferta de suelo de menor valor, promueve intervenciones de gran escala (atendiendo a la lógica empresarial) y disminuye las dimensiones de los espacios habitables a umbrales críticos (situación estructural para sectores que no cuentan con capacidad de ahorro que les permita ampliar la vivienda). Como consecuencia existen efectos directos en la población de escasos recursos relocalizada: la dificultad para afrontar los costos de transporte y movilidad urbana contribuye al aislamiento social, se desestructuran las redes laborales y sociales y la mayor dificultad para acceder a oportunidades de trabajo (dado que los nuevos territorios de la política habitacional son homogéneamente pobres) contribuye a la reproducción de la pobreza.

Las nuevas formas de segregación residencial socio-económica derivadas de la política habitacional llevan a interrogarnos cuáles serían las posibles líneas de acción para atenuar estos procesos. Sin duda que se debería considerar una menor escala en las intervenciones residenciales (menor cantidad de unidades por programa) y una mayor participación de los beneficiarios y sus demandas en el diseño de las unidades habitacionales.

Frente al criterio de vivienda terminada como línea prioritaria de intervención, se deberían promover programas de regularización y mejoramiento de barrios, desarrollar propuestas de densificación predial para facilitar el acceso al suelo e intervenir en los tejidos residenciales existentes contribuyendo a su revitalización poblacional y optimizando la capacidad de la estructura urbana instalada en la ciudad construida.

La disociación entre políticas habitacionales y políticas de desarrollo urbano debe ser superada con una visión integral de la planificación física del territorio. El acceso al suelo urbano es sin duda el tema crítico que atraviesa esta problemática. Considerando las implicancias económicas, sociales y físico-espaciales derivadas de la materialización de los conjuntos de vivienda social subsidiada en los bordes urbanos, y sus efectos en la segregación residencial y en la extensión urbana, es posible pensar en opciones que alienten la diversidad programática, consideren otras formas de tenencia (no solo la vivienda en propiedad) y promuevan la mixtura social para responder a los nuevos desafíos de alcanzar ciudades más integradas.

\section{Bibliografía}

ABRAM0, Pedro. La teoría económica de la favela: cuatro notas sobre la localización residencial. [En línea] Ciudad y territorio: Estudios territoriales. (136-137): 273-294, 2003. ISSN 1133-4762. Disponible en: http://dialnet.unirioja.es/servlet/ articulo? codigo $=819540$. 
ANGEL, Shlomo; PARENT, Jason; CIVCO, Daniel y BLEI, Alejandro The persistent decline in urban densities: global and historical evidence of 'sprawl'. [En línea]. Lincoln Institute of Land Policy. Julio 2010. [Fecha de consulta: 28 enero 2015]. Disponible en: https://www.lincolninst.edu/pubs/download. asp?doc_id=1085\&pub_id=1834.

ARRIAGADA, Camilo y RODRÍGUEZ, Jorge. Segregación residencial en áreas metropolitanas de América Latina: magnitud, características, evolución e implicaciones de política. Santiago, CEPAL. 2003. Serie Población y Desarrollo, 47. ISBN 9213222734.

BONDUKI, Nabil. Avances, limitaciones y desafíos de la política habitacional del gobierno de Lula: derecho a la vivienda en oposición al derecho a la ciudad. En: BOLÍVAR, Teolinda, comp.; GUERRERO, Mildred, comp. y RODRÍGUEZ, Marcelo, comp. Casas de infinitas privaciones ¿Germen de ciudades para todos? Quito, CLACSO. 2014. p. 51-93. ISBN: 978-9942-09-189-5.

BOURDIEU, Pierre. Las estructuras sociales de economía. Buenos Aires, Argentina, Manantial. 2001. 271 p.

BRAIN, Isabel y SABATINI, Francisco. Los precios del suelo en alza carcomen el subsidio habitacional, contribuyendo al deterioro en la calidad y localización de la vivienda social. ProUrbana. (4): 2-13, mayo 2006.

BORJA, Jordi. Revolución y contrarrevolución en la ciudad global: las expectativas frustradas por la globalización de nuestras ciudades. [En línea].
EURE. 33(100): 35-50, 2007. ISSN 0250-7161. DOI 10.4067/S0250-71612007000300003.

CLICHEVSKY, Nora. Informalidad y segregación urbana en América Latina. Una aproximación. Santiago, CEPAL. 2000. Serie Medio Ambiente y Desarrollo nro. 28.

CUENYA, Beatriz. Políticas habitacionales inclusivas. En: BARRETO, Miguel y LENTINI, Mercedes. Hacia una política integral del hábitat. Aportes para un observatorio de política habitacional en Argentina. Buenos Aires, Café de las Ciudades. 2014. p. 95-111. ISBN 978-987-3627-01-9.

--- Descentralización y política de vivienda en Argentina. En: CUENYA, Beatriz y FALU, Ana. Reestructuración del Estado y política de vivienda en Argentina. Buenos Aires. CEA, Oficina de Publicaciones del CBC, 1997.

DAVIS, Mike. Planeta de ciudades miseria. Madrid, FOCA. 2006. 284 p. ISBN 978-84-95440-96-9.

DI VIRGILIO, María Mercedes y PERELMAN, Mariano. Ciudades latinoamericanas. La producción social de las desigualdades urbanas. En: DI VIRGILIO, María Mercedes, coord. y PERELMAN, Mariano, coord. Ciudades latinoamericanas. Desigualdad, segregación y tolerancia. Buenos Aires, CLACSO. 2014. P. 8-19. ISBN 978-987-722-019-3.

DUHAU, Emilio. La división social del espacio metropolitano. Una propuesta de análisis. [En línea]. Nueva Sociedad. (243): 79-91, enero 2013. ISSN: 0251-3552. Disponible en: http://nuso.org/media/ articles/downloads/3917_1.pdf. 
ELORZA, Ana Laura. Estrategias de reproducción social de familias relocalizadas, entre la adaptación y el cambio. Conciencia Social. (12): 53-59, diciembre, 2008.

\section{ESQUIVEL, María Teresa; MAYA, Esther y CERVAN-}

TES, Jorge. La promoción privada y los grandes conjuntos habitacionales: nuevas modalidades de acceso a la vivienda. [En línea] Scripta Nova. 9(194 / 21), 2005. ISSN 1138-9788. Disponible en: http://www.ub.edu/geocrit/sn/sn-194-21.htm.

FERNÁNDEZ WAGNER, Raúl. El sistema de la vivienda pública en Argentina. Revisión desde la perspectiva de los regímenes de vivienda. En: BARRETO, Miguel y LENTINI, Mercedes. Hacia una política integral del hábitat. Aportes para un observatorio de política habitacional en Argentina. Buenos Aires, Café de las Ciudades. P. 27-94. 2014. ISBN 978-987-3627-01-9.

HARVEY, David. Mundos urbanos posibles. En: RAMOS, Ángel Martín. Lo urbano en 20 autores contemporáneos. Barcelona, Universidad Politécnica de Catalunya. 2000. P. 118-132. ISBN 978-848-301-752-4.

HIDALGO, Rodrigo. ¿Se acabó el suelo en la gran ciudad? Las nuevas periferias metropolitanas de la vivienda social en Santiago de Chile. [En línea] Eure. 33(98): 57-75, 2007. ISSN 0250-7161. DOI 10.4067/S0250-71612007000100004.

KATZMAN, Rubén. Seducidos y abandonados: el aislamiento social de los pobres urbanos. [En línea].
Revista CEPAL. (75): 171-189, diciembre 2001. ISSN 1682-0908. Disponible en: http://www. cepal.org/publicaciones/xml/6/19326/Katzman. pdf.

MARENG0, Cecilia. Urban sprawl and planning: Confronting the challenges in a context of social inequity. Case study: Córdoba - Argentina. The Netherlands, International Forum on Urbanism (IFoU). 2008. Tesis doctoral.

--- Una aproximación a la segregación residencial como punto de partida en la formulación de políticas. Revista INVI. 19(50): 165-181, 2004. ISSN 0716-5668.

MARENGO, Cecilia y ELORZA, Ana Laura. Tendencias de segregación residencial socioeconómica: el caso de Córdoba (Argentina) en el período 20012008. EURE. 40(120): 111-133, 2014. ISSN 02507161. DOI 10.4067/S0250-71612014000200006.

MATTOS, Carlos de. Una nueva geografía latinoamericana en el tránsito de la planificación a la gobernanza, del desarrollo al crecimiento. EURE. 36 (108): 167-179, agosto 2010. ISSN 0250-7161. DOI 10.4067/S0250-71612010000200010.

MOLINATTI, Florencia. Segregación residencial socioeconómica en la ciudad de Córdoba (Argentina): Tendencias y patrones espaciales. Revista INVI. 28(79): 61-94, 2013. ISSN 0718-8358. DOI 10.4067/S0718-83582013000300003.

MONAYAR, Virginia. Informalidad urbana y acceso al suelo. Políticas habitacionales en el municipio de Córdoba 1990-2010. Tesis Doctoral en Ciencias 
Sociales, orientación Geografía, marzo 2014. Sin publicar.

ONU HABITAT. Estado de las ciudades en América Latina y el Caribe 2012. Rumbo a una nueva transición urbana. [En línea]. Brasil, Programa de las Naciones Unidas para los Asentamientos Humanos, ONU-Hábitat. 2012. [Fecha de consulta: 5 enero 2013]. Disponible en: http://www.onuhabitat. org/index.php?option=com_docman\&task $=$ doc details\&gid=816\&Itemid $=538$.

PRADILLA COBOS, Emilio. Capital, Estado y vivienda en América Latina. México, Fontamara. 1987. 308 p. ISBN 968476037X.

PROGRAMA de Naciones Unidas para el Desarrollo. Aportes para el desarrollo humano en Argentina / 2009: segregación residencial en Argentina. [En línea]. PNUD Argentina. 2009. [Fecha de consulta: 12 febrero 2010] Disponible en: http://www. ar.undp.org/content/argentina/es/home/library/ human_development/AportesDHArg2.html.

RODULF0, María Beatriz. Políticas habitacionales. Alternativas de intervención y gestión. En: Seminario iberoamericano ciencia y tecnología para el hábitat popular. Construcción y participación del conocimiento $\left(1^{\circ}, 2006\right.$, Córdoba). Ciencia y tecnología para el hábitat popular. Buenos Aires, Nobuko. 2007. ISBN 978-987-584-077-5.

RODRÍGUEZ, Alfredo y SUGRANYES, Ana. Los con techo. Un desafío para la política de la vivienda social. Santiago, Chile, Ediciones Sur. 2005. 267 p. ISBN 956-208-074-9.
RODRÍGUEZ, Inés. Vivienda social latinoamericana: la clonación del paisaje de la exclusión. [En línea] ACE Arquitectura, Ciudad y Entorno. 1(2): 2055, octubre 2006. ISSN 1887-7052. Disponible en: http://revistes.upc.edu/ojs/index.php/ACE/ article/view/2342.

RODRÍGUEZ, Jorge. Segregación residencial socioeconómica: ¿qué es?, ¿cómo se mide?, ¿qué está pasando?, ¿importa? [En línea]. CEPAL / ECLAC. Agosto, 2001. ISBN: 92-1-321882-6. [Fecha de consulta: 18 mayo 2007]. Disponible en: http:// www.cepal.org/es/publicaciones/7149-segregacion-residencial-socioeconomica-que-es-comose-mide-que-esta-pasando.

RUSK, David. Urban growth. Conversations on urban policy: the USA and the Netherland. En: RUSK, David. Planning Metropolis. Urban growth and social patterns. Amsterdam, Deltametropool. 1999. P. 7-32. ISBN 90-76630-02-0.

SABATINI, Francisco. Medición de la segregación residencial: reflexiones metodológicas desde la ciudad latinoamericana. En: SABATINI, Francisco, ed. y CÁCERES, Gonzalo, ed. Barrios cerrados en Santiago de Chile: entre la exclusión y la integración social. Santiago, Lincoln Institute of Land Policy: Instituto de Geografía, Pontificia Universidad Católica de Chile, 2004. ISBN 9562992284.

SABATINI, Francisco; CÁCERES, Gonzalo y CERDA, Jorge. Segregación residencial en las principales ciudades chilenas: Tendencias de las tres últimas décadas y posibles cursos de acción. [En línea]. 
EURE. 27(82): 21-42, 2001. ISSN 0250-7161. DOI 10.4067/S0250-71612001008200002.

SARAVI, Gonzalo. Mundos aislados: segregación urbana y desigualdad en la ciudad de México. [En línea]. Eure. 34(103): 93-110, 2008. ISSN 02507161. DOI 10.4067/S0250-71612008000300005.

TECCO, Claudio y FERNÁNDEZ, Silvana. Políticas públicas y segregación residencial socioeconómica en la ciudad de Córdoba, Argentina. [En línea] Biblioteca Digital Municipal. 2005. [25 de septiembre 2015]. Disponible en: http://biblioteca.municipios.unq.edu.ar/modules/mislibros/archivos/polpubysegregacionresidencial2005teccofernandez. pdf.

YUJNOVSKY, Óscar. Sectores populares y política estatal de vivienda (Argentina 1976-1981). En: Ciudades y sistemas urbanos: economía informal y desorden espacial. Buenos Aires, CLACSO. 1984. P. 183-206. ISBN 950923108X. 